\title{
Construction of a photothermal Venus flytrap from conductive polymer bimorphs
}

\author{
Hanwhuy Lim, Teahoon Park, Jongbeom Na, Chihyun Park, Byeonggwan Kim and Eunkyoung Kim
}

A photothermally foldable soft bimorph was prepared via the dry transfer of poly(3,4-ethylenedioxythiophene) (PEDOT) doped with tosylate onto a poly(dimethylsiloxane) film. The photothermal folding was optimized via reversible actuation by controlling the thickness of each layer and the temperature increase to afford large deflection and displacement up to $150^{\circ}$ and $>20 \mathrm{~mm}$, respectively, upon exposure to near-infrared (NIR) light $(808 \mathrm{~nm})$. A two-dimensional array of the bimorph converted into complex three-dimensional architectures, such as a Venus flytrap, under light and reversibly unfolded in the dark. Taking advantage of the photothermal nature of PEDOT, a localized heat pocket was generated inside the folding structure. Thus, a Venus flytrap with a hot pocket reaching $100{ }^{\circ} \mathrm{C}$ was realized for the first time. The Venus flytrap could trap and move an object within a few seconds of NIR exposure.

NPG Asia Materials (2017) 9, e399; doi:10.1038/am.2017.101; published online 7 July 2017

\section{INTRODUCTION}

There is growing interest in the design of micro-robots or biomimetic micro-motors that mimic biological systems with life-like motions in response to external stimuli, such as heat, light or moisture. ${ }^{1-6}$ Among them, photothermal conversion has attracted increasing attention as a method for creating mechanical motion because of the opportunity for wireless control, low noise, programmability and spatial localization of the mechanical motion. ${ }^{7-10}$ In particular, near-infrared (NIR) light can penetrate biological tissues to remotely actuate micro-robots inside bodies, which could lead to applications for micro-motors in vivo.

Conductive polymers (CPs), such as polypyrrole, polyaniline and poly(3,4-ethylenedioxythiophene) (PEDOT), have been used to provide various actuations due to their high electrical conductivity, sorption/desorption properties and flexibility. ${ }^{11-14}$ Specifically, significant efforts have been focused on $\mathrm{CP}$ actuators that exhibit large volume changes in air upon the sorption or desorption of the water vapor present in ambient air under the application of an electric current. ${ }^{15-20}$ For example, a humidity-driven, bilayered, anisotropic actuator has been reported based on a PEDOT-polystyrenesulfonic acid (PEDOT:PSS) layer with a soft poly(dimethylsiloxane) (PDMS) layer. This actuator showed an intrinsic sensing capability via the joule effect or directly via environmental humidity variations. ${ }^{11}$ However, photothermal CPs with reversible folding to generate threedimensional (3D) structures or heat pockets have not been previously reported. This can be attributed to the significant challenges present in the selection of materials and fabrication of a soft platform based on photothermal CPs. The photothermal properties of CPs have been intensely investigated only in the fields of photothermal energy harvesting and cell engineering. ${ }^{21-26}$ Therefore, the application of highly photothermal CPs, such as PEDOTs, into foldable bimorphs is an interesting challenge. The other challenge is the fabrication of bimorphs with CPs, which involves the transfer of a CP layer onto a soft layer. The well-known, water-assisted, transfer-printing methods for fabricating conventional soft actuators are incompatible with water-permeable CPs, such as PEDOT-poly(styrenesulfonate). ${ }^{27}$ Moreover, the hydrophilic solvents used for a wet transfer process could change the doping state of the pristine PEDOT films. Therefore, a dry transfer method could be an alternative to the conventional wet transfer method. Recently, a highly conductive PEDOT film was obtained from a one-step solution casting polymerization of ethylenedioxythiophene (EDOT) in the presence of ion tosylate, pyridine and poly(ethylene glycol)-block-poly(propylene glycol)-block-poly (ethylene glycol). The resultant PEDOTs have a hydrophobic polymer surface, are water insoluble and have better surface energy matching with a hydrophobic surface than conventional PEDOT:PSSs. ${ }^{28,29}$ Furthermore, the PEDOTs with tosylate dopants have high photothermal properties in a thin-film state (sub-micron level). ${ }^{24,25}$ Such one-step film preparation from EDOT and the high electrical conductivity plus photothermal properties of the film could be advantages for PEDOT as an active bimorph over other materials. $^{7,30,31}$ To transfer the PEDOT layer directly onto a soft PDMS layer, a dry transfer method could be explored and used to take advantage of the curing-induced detachment properties of the PDMS surface. $^{32,33}$ This dry transfer method would be essential to maintain the doping state of the PEDOT layer because the method does not use a solvent or any additives.

Herein we report on a photothermally foldable bimorph composed of dry-transferred PEDOT on a PDMS film. The polymer bimorphs take advantage of the high-photothermal properties of PEDOT to deliver large volume changes in a rubber layer, which has high-elastic 
property and low Young's modulus, using heat generated via NIR exposure. ${ }^{34-37}$ Therefore, the bimorph can function as a foldable sheet and produce large deflections and displacements along with heat build-up upon exposure to an NIR source. The movement and displacement of the bimorph can be remotely controlled via radiation from a NIR laser or sunlight. In addition to the photothermal folding, the programmable bimorph can generate a complex $3 \mathrm{D}$ architecture such as a Venus flytrap, which can be useful for a broad range of applications.

\section{MATERIALS AND METHODS}

\section{Materials}

A Si wafer made up of $200 \mathrm{~nm}$-thick silicon dioxide was purchased from Buysemi, Korea, and was used as the substrate for the PEDOT coating. Iron (III) chloride hexahydrate (purity 97\%); $p$-toluenesulfonic acid monohydrate (purity >98.5\%); poly(ethylene glycol)-block-poly(propylene glycol)-blockpoly(ethylene glycol) with an average molecular weight of $2800 \mathrm{Da}$; 3,4ethylenedioxythiophene (purity 97\%); pyridine (purity 99.8\%); anhydrous methanol (purity 99.8\%); and $n$-butanol (purity 99.8\%) were purchased from Aldrich Chemicals. The Sylgard 184 kit was purchased from (Dow Corning, MI, USA). Iron (III) $p$-toluenesulfonate hexahydrate was purchased from Sigma Aldrich Chemicals, St Louis, MO, USA. It was dehydrated at $125^{\circ} \mathrm{C}$ for $3 \mathrm{~h}$, and the resultant lump was crushed into a fine powder. After further evaporation of the water, the residual water was completely removed via extended drying in a vacuum oven. Then, the dried, fine powder of iron (III) tosylate (FeTos) was dissolved in $\mathrm{BuOH}$ to create a $40 \mathrm{wt} \%$ solution. The solution was stirred at $45^{\circ} \mathrm{C}$ for $6 \mathrm{~h}$ and filtered through a polytetrafluoroethylene syringe filter $(0.45 \mu \mathrm{m}$ pore size, $12 \mathrm{~mm}$ diameter).

\section{Preparation of PEDOT films on a Si wafer}

$\mathrm{Si}$ wafers were pre-cleaned with ethanol. A solution of $40 \mathrm{wt} \%$ of FeTos in $n$-butanol $(1 \mathrm{~g})$ was mixed with pyridine $(13.5 \mathrm{mg})$ and the poly(ethylene glycol)-block-poly(propylene glycol)-block-poly(ethylene glycol) triblock copolymer $(200 \mathrm{mg})$. EDOT was then added to the solution to provide a molar ratio of pyridine:FeTos:EDOT $=0.55: 2.25: 1$. The solution was spin-coated at 1500 r.p.m. for $30 \mathrm{~s}$, and the coated layer was heated to $60^{\circ} \mathrm{C}$ for $1 \mathrm{~h}$ to afford PEDOT doped with tosylate (PP-PEDOT). The PP-PEDOT-coated Si wafers were washed with ethanol to remove any residual oxidant, low-molecularweight oligomers and impurities. The PP-PEDOT film thickness was $130 \mathrm{~nm}$, which was determined using an Alpha-step profilometer (Tencor Instruments, Milpitas, CA, USA), and the thickness increased to 290 and $400 \mathrm{~nm}$ upon repeating the solution casting polymerization.

\section{Preparation of soft bimorph actuators}

The PDMS prepolymer solution was prepared from Sylgard 184 by mixing the base resin and the curing agent at a 10:1 ratio by weight. After degassing in a vacuum for $5 \mathrm{~min}$, the solution was spin-coated onto the PP-PEDOT surface of a Si wafer at spin rates of 300, 500 and 1000 r.p.m. for $1 \mathrm{~min}$ and cured at $70{ }^{\circ} \mathrm{C}$ in a drying oven for $12 \mathrm{~h}$ to produce 300,160 and $70 \mu \mathrm{m}$-thick films, respectively, which were homogeneous and highly adhesive on the PPPEDOT layer. Controlling the thickness of the PDMS layer via the spincoating speed was consistent with previous work. ${ }^{38,39}$

After curing the top PDMS layer, the adhesion of the PP-PEDOT layer on the $\mathrm{Si}$ wafer became weaker compared to the uncured sample due to the increase in the overall free energy during the polymerization of the PDMS presolution. ${ }^{40,41}$ Thus, the PP-PEDOT layer with the PDMS top coat was easily detached from the Si wafer. Hence, the dry transfers simply pulled the PDMScoated, PP-PEDOT layer from the Si wafer in the air. The PP-PEDOT layer also cleanly detached from the Si wafer via a wet transfer process using water to provide a PDMS/PEDOT bimorph. However, only the dry-transferred PPPEDOTs were used in this paper for the actuator and folding experiments to prevent any negative effects from polar solvents.

The geometry of the bimorph actuator upon bending was similar to that of previous bending actuators. One end of the bimorph film was fixed and the other end (the free end) could move freely upon exposure to a NIR laser. The bending actuation performance of the bimorph was determined using the laser intensity and thickness of the layers in the bimorph. Other actuators for wireless movement in air or water were also designed as shown in the figures and movie. The two-dimensional (2D) array structure was prepared by cutting the samples transferred from the Si wafer. The size of the bimorph strips for the photothermal actuation characterization was $5 \times 10 \mathrm{~mm}^{2}$. The sizes of the strips for the worm-like movement and water floating fish actuator were $25 \times 3 \mathrm{~mm}^{2}$ and $18 \mathrm{~mm}$ (length) $\times 6 \mathrm{~mm}$ (center width), respectively. Two types of sunflower-like, 2D array (Figure $4 \mathrm{~b}$ ) were prepared to form an excavator (13 lobes) and Venus flytrap (16 lobes) with sizes $\left(L_{1}, L_{2}\right.$ and $\left.L_{3}\right)$ of $12 \mathrm{~mm}$, $4 \mathrm{~mm}, 0.8 \mathrm{~mm}$ and $17 \mathrm{~mm}, 6 \mathrm{~mm}, 1 \mathrm{~mm}$, respectively. We specified the two types as sunflower A and B, respectively. The dry transfer method is scalable and practical for transferring large-area, thin-film electronic devices because detaching PP-PEDOT via PDMS curing is independent of the surface area. The area of the PP-PEDOT/PDMS bimorphs was as large as $10 \times 10 \mathrm{~cm}^{2}$, and they were cut into strips $\left(5 \times 10 \mathrm{~mm}^{2}\right.$ for bimorph characterization) or biomimetic structures. Thus, the dry transfer method was very useful for the preparation of non-Si-based bimorphs and flexible electronic devices.

\section{Characterization and instrumentation}

Sample thickness was determined using a thickness profiler ID-C112 (Mitutoyo Corp., Kanagawa, Japan) and Alpha-step profilometer (Alpha-step IQ). The actuator fabrication uniformity was examined using ultraviolet/visible NIR absorption spectroscopy (PerkinElmer Lambda 750, Waltham, MA, USA). The mechanical properties of the polymer films were measured using a Universal Testing Machine (Yeonjin TopTac 2000, Yeonjin, Gangseo-gu, Seoul, Korea). To determine the sensitivity of the bimorphs for photothermal actuation, $5 \times 10 \mathrm{~mm}^{2}$ samples were irradiated with different intensities using an $808 \mathrm{~nm}$ NIR laser (B\&W Tek, Inc., Newark, DE, USA) from 13-198 mW at a distance of $7 \mathrm{~cm}$. The NIR source used to actuate movement in the large samples (microfish and sunflowers) was a $0.253-1.1 \mathrm{~W}$ NIR coherent diode laser (808 nm, DPSS laser series, Lasos, Franz-Loewen-Str., Jena, Germany). The samples were vertically irradiated from the surface at a distance of $10 \mathrm{~cm}$. The photographic images and movie were obtained from the Dino lite (Narae Plus Co., Ltd, Gangnam-gu, Seoul, Korea) used to record the photothermal movement. The photothermal temperature rise and thermal images were obtained using infrared cameras from FLIR (E40, FLIR Systems, Inc., Wilsonville, OR, USA) and Testo (875 i Testo, Inc., Lenzkirch, Germany). The conductivities of the pristine PP-PEDOT film and the bimorph were determined with the four-point probe method (Napson, RT-70V/RG-5, Napson Corp., Chiba, Japan) for a thin film using the equation below ${ }^{42}$

$$
\sigma_{\mathrm{s}}=\frac{\ln 2}{\pi \times R \times T}
$$

where $\sigma_{\mathrm{s}}$ is the electrical conductivity, $R$ is the sheet resistance and $T$ is the thickness of the PP-PEDOT layer. The electrical conductivities of the pristine PP-PEDOT on a Si wafer (before dry transfer) and on PDMS (after transfer) were measured three times for each sample, and the average values are listed in Supplementary Table S1.

\section{RESULTS AND DISCUSSION}

\section{Design and fabrication of the PP-PEDOT/PDMS bimorphs}

The bimorph configuration composed of a photothermal PEDOT layer with a soft PDMS layer was prepared via the dry transfer of the PEDOT layer to a soft PDMS layer, as illustrated schematically in Figure 1a. The PEDOT layer was initially prepared on a flat, defect-free $\mathrm{Si}$ wafer via solution casting polymerization of EDOT in the presence of FeTos, pyridine and poly(ethylene glycol)-block-poly(propylene glycol)-block-poly(ethylene glycol). The prepared PP-PEDOT film, abbreviated as PP-PEDOT here after, was uniformly coated on a $\mathrm{Si}$ wafer, as shown in the cross-section scanning electron microscopy image (Figure 1b,i). A mixture of the liquid PDMS prepolymer solution was coated onto the PP-PEDOT surface (Figure 1a(iii)). The PDMS-coated PP-PEDOT layer was detached from the Si wafer by lifting the film border. The thicknesses of the detached bilayer films of 
a

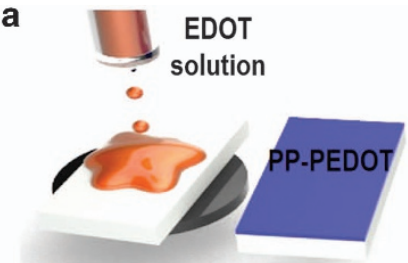

i. Spin-coating

ii. Heating

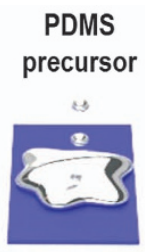

iii. Spin-coating

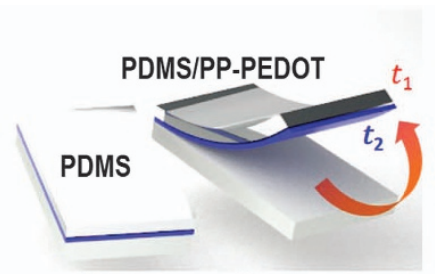

iv. Heating vry transfer

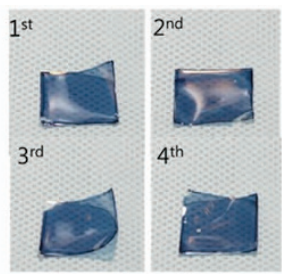

vi. Transferred bilayer

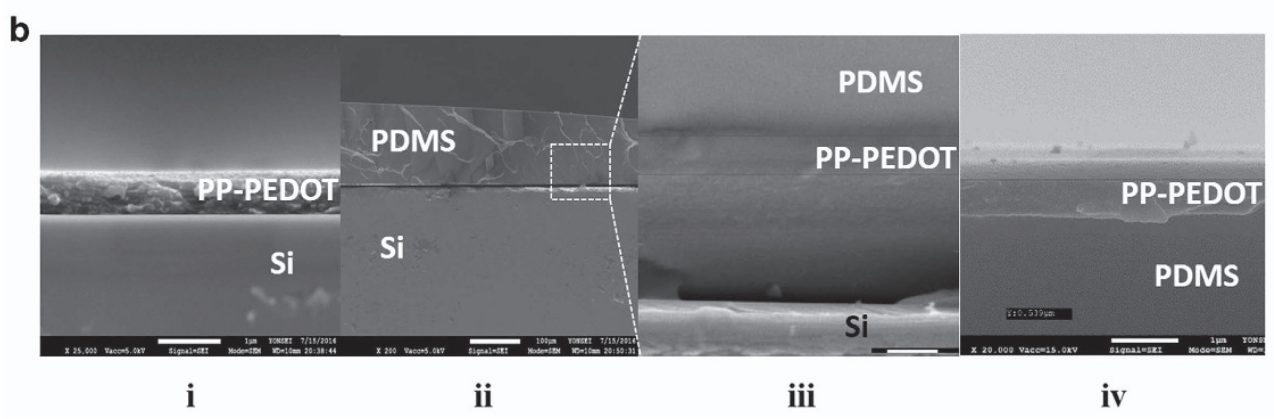

Figure 1 (a) Schematic diagram of the dry transfer process of the PP-PEDOT layer onto a soft poly(dimethylsiloxane) (PDMS) layer. (i,ii) Coating of PPPEDOT on a Si wafer via solution casting polymerization of ethylenedioxythiophene (EDOT). (iii, iv) Coating of the PDMS layer on the PP-PEDOT layer. (v) Dry transfer of the PP-PEDOT layer onto the PDMS layer. (vi) Photographic image of the bimorphs of PP-PEDOT (130 nm) on PDMS (160 $\mu \mathrm{m})$ prepared via multiple dry transfer processes from the first to the fourth transfer. (b) Crosscut scanning electron microscope images of the (i) PP-PEDOT coated on a Si wafer, (ii) PDMS/PP-PEDOT/Si layer, (iii) enlarged image of ii and (iv) transferred bimorph of PP-PEDOT and PDMS. PEDOT, poly(3,4-ethylenedioxythiophene).

PDMS $\left(t_{1}\right)$ and PP-PEDOT $\left(t_{2}\right)$ were consistent with the thicknesses measured before detachment (Figure $1 \mathrm{~b}$ ). The interfacial gap between the PP-PEDOT layer and the Si wafer increased in the presence of the PDMS layer (Figure 1b(ii) and (iii)) compared to the gap without PDMS (Figure $1 \mathrm{~b}(\mathrm{i})$ ). This could be attributed to curing the PDMS precursor on top of the PP-PEDOT surface. After curing, the polymerization generated a large surface energy, which may result in a large gap between the PP-PEDOT layer and the Si wafer. Thus, the PP-PEDOT layer could be transferred onto the PDMS layer, which was similarly observed for the transfer of the active layer via the PDMS stamp. ${ }^{33}$ Figure $1 \mathrm{~b}(\mathrm{iv})$ shows the tight adhesion of PP-PEDOT onto the PDMS layers after it was detached from the Si wafer.

The dry transfer of PP-PEDOT was performed at room temperature, which is much lower than the decomposition temperature of PPPEDOT, and no solvent was used. Thus, the transferred PP-PEDOT layer remained in its pristine state, without de-doping or decomposition. Supplementary Figure S1 shows the absorption spectrum of the PP-PEDOT layer on the PDMS surface obtained using the dry transfer method. The film showed the same absorption spectrum as the pristine PP-PEDOT (black square line) coated on a slide glass.

The Si surface after the PP-PEDOT transfer was clean and defectfree, without any organic residue. Therefore, it could be reused for repeated dry transfer processes. The Si surface used for the first PPPEDOT transfer was recoated with a fresh PP-PEDOT layer and transferred to another PDMS layer. This process was repeated several times. The second to fourth PP-PEDOT films transferred showed the same absorption spectra as the first PP-PEDOT transferred. The absorbances at 800 and $1600 \mathrm{~nm}$, which are characteristic of the presence of polarons and bipolarons, respectively, indicated that the doping state of PP-PEDOT was the same for all the samples, including the pristine PP-PEDOT film. The transparency of the PDMS-coated PP-PEDOT layer from the multiple transfer process was almost the same as that of the pristine PP-PEDOT on a glass substrate (Supplementary Figure S1a). Supplementary Figure S1b shows the absorbance for the PP-PEDOT bimorphs at 400-1600 nm. To examine the electrical change in the pristine PP-PEDOT film after the dry transfer process, the electrical conductivities of the pristine PPPEDOT film on a Si wafer and PDMS film (bimorph) were determined using the four-point probe method for a thin film. ${ }^{42}$ The electrical conductivities increased as the PP-PEDOT thickness increased, and the maximum conductivity was $1000 \mathrm{~S} \mathrm{~cm}^{-1}$, which is similar to the value in our previous report. ${ }^{43}$ Importantly, the conductivity difference $\left(\Delta \sigma_{\text {ave }}\right)$ between PP-PEDOT on a Si wafer (before dry transfer) and on PDMS (after transfer) was small, as shown in Supplementary Table S1. This result indicates that the pristine state of the PP-PEDOT film is well preserved after the dry transfer to PDMS. Furthermore, the dry transfer method is scalable and practical for transferring large-area, thin-film electronic devices because detaching PP-PEDOT via PDMS curing is independent of the surface area. The areas of the PP-PEDOT/PDMS bimorphs were as large as $10 \times 10 \mathrm{~cm}^{2}$. The bimorphs were cut into strips $\left(5 \times 10 \mathrm{~mm}^{2}\right.$ for bimorph characterization) or biomimetic structures. The dry transfer method was very useful for preparation of non-Si-based bimorphs and flexible electronic devices.

\section{Photothermal properties and thermal actuation simulation}

As shown in Figure 2a, the PEDOT/PDMS bimorphs absorb NIR light and reach an absorbance of 1 with a thick film $\left(t_{2}=400 \mathrm{~nm}\right)$ in both the NIR-1 (750-1000 nm) and NIR 2 regions $(1000-1700 \mathrm{~nm}) .{ }^{44,45}$ Because of the large absorption, the PP-PEDOT surface was heated upon exposure to a NIR light, as shown in the thermal image in Figure 2c. The maximum temperature $\left(T_{\max }\right)$ of the PP-PEDOT surface on a PDMS $\left(t_{1}=70 \mu \mathrm{m}\right)$ was 96,117 and $131^{\circ} \mathrm{C}$, for $t_{2}$ of 130 , 290 and $400 \mathrm{~nm}$, respectively, upon exposure to a $198 \mathrm{~mW}$ NIR source for $10 \mathrm{~s}$ (Figure $2 \mathrm{~b}$ and $\mathrm{c}$ ). The photothermal temperature rise linearly correlated to the input laser intensity $\left(I_{0}\right)$ with a slope of $0.32-0.38 \mathrm{~K} \mathrm{~mW}^{-1}$, as shown in Supplementary Figure S2a. 
a

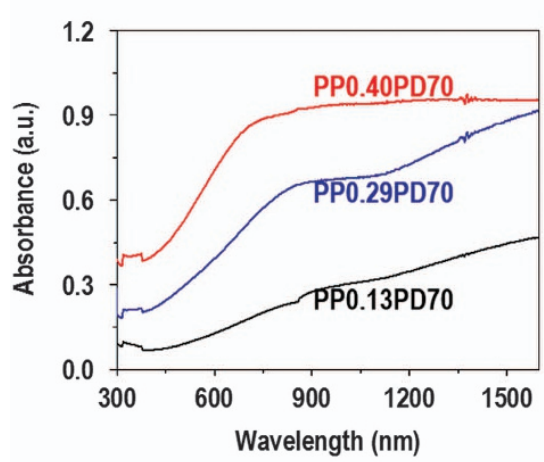

d

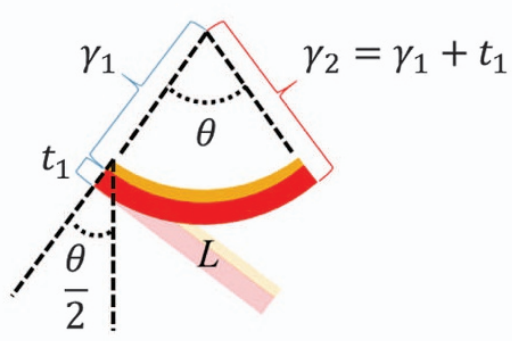

b

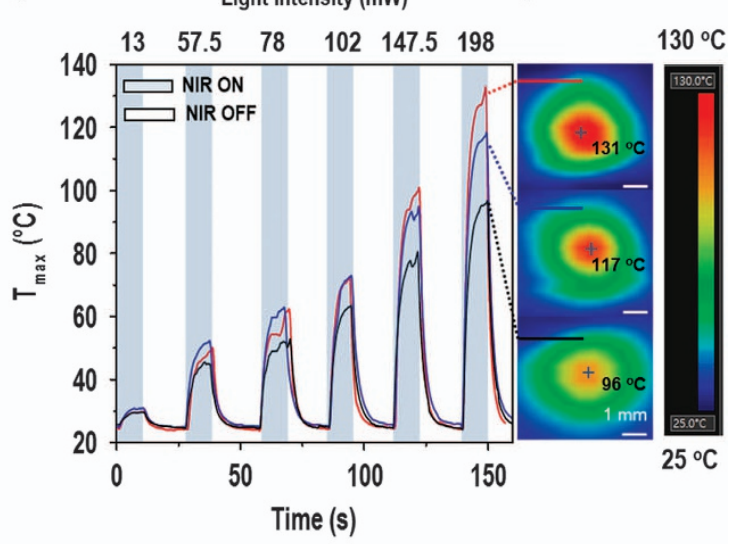

e

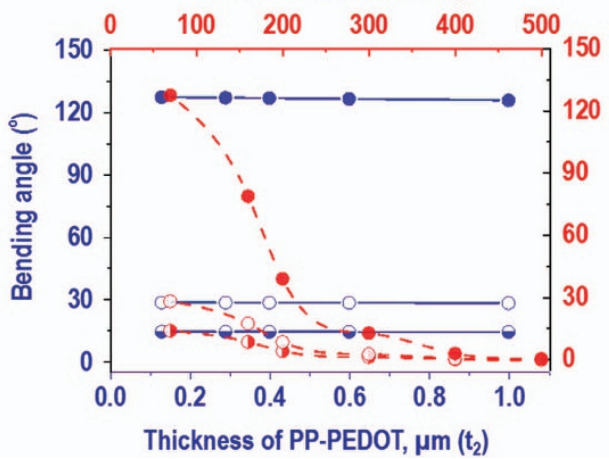

Figure 2 (a) Ultraviolet/visible spectra of PP-PEDOT/PDMS bimorphs with different thicknesses of the PP-PEDOT layer (red, blue and black for 400, 290 and $130 \mathrm{~nm}$, respectively) on a $70 \mu \mathrm{m}$-thick poly(dimethylsiloxane) (PDMS) layer. (b) Temperature increase in the bimorphs (a) upon near-infrared (NIR) exposure for $10 \mathrm{~s}$ with different NIR intensities as given. (c) Thermal image of the bimorphs (a) under NIR exposure for $10 \mathrm{~s}$ at $198 \mathrm{~mW}$ : From top to bottom, PP0.40PD70, PP0.29PD70 and PP0.13PD70. (d) Model for thermal actuation of a bimorph, where $r, \theta, t_{1}, L$ are the radius of curvature, bending angle, thickness of the PDMS layer and length of the bimorph, respectively. (e) Simulation on the bending angle of a bimorph with different thicknesses of PPPEDOT (blue) and PDMS (red) at temperature differences of 90 (filled circle), 20 (open circle) and $10 \mathrm{~K}$ (half-filled circle), according to equation (5) using $\mathrm{CTE}=1 \times 10^{-4}$ and $3.14 \times 10^{-4} \mathrm{~K}^{-1}$ and Young's modulus $E=2 \mathrm{GPa}$ and $0.79 \mathrm{MPa}$ for PP-PEDOT and PDMS, respectively. PEDOT, poly(3,4ethylenedioxythiophene).

At the same time, the bimorph films responded to the NIR light by folding. The folding results in different angles and displacements depending on the laser power and exposure direction. PDMS is an elastomer with a large coefficient of thermal expansion (CTE), $3.14 \times 10^{-4} \mathrm{~K}^{-1}, 46$ whereas the CTE of PP-PEDOT is $0.8-1.2 \times 10^{-4} \mathrm{~K}^{-1}$. ${ }^{4}$ The thermal actuators were affected by the difference in the CTE between the layers, and the PDMS layer expanded three to four times more than the PP-PEDOT layer with the photothermally induced heat. As a result, the bimorph strip showed a visible displacement and deflection upon exposure to a NIR laser.

In the bimorph actuators, each layer expands at different rates according to

$$
\Delta L_{i}=\alpha_{i} \times L \times\left(T-T_{0}\right)
$$

where $\Delta L_{i}$ is the linear thermal expansion of the $i$ th layer, $L$ is the length of the actuator, $T$ is the actuation temperature, $\alpha_{i}$ is the CTE of the $i$ th layer and $T_{0}$ is the initial temperature. Because of the different CTEs, the materials expand at different rates, producing a bend in the strip in the direction of the material with the lower CTE (PP-PEDOT). Furthermore, the Young's modulus of the layer materials is also important for the actuation. ${ }^{48-50}$ The Young's modulus of the PDMS film, which was determined from the stress-strain curves
(Supplementary Figure S2b) of the PDMS films, was 0.79 and $0.86 \mathrm{MPa}$ for the films with a thickness of 70 and $300 \mu \mathrm{m}$, respectively. The Young's modulus of PDMS matched well with the reported value. ${ }^{51}$ The stress-strain curves for a bimorph of PP0.13PD300 were reversible up to a $30 \%$ strain. From the slope of the stress-strain curve, the Young's modulus of PP-PEDOT was determined to be $2 \mathrm{GPa}$, which agreed with the reported values of $1.3-2.6 \mathrm{GPa}^{34-37}$

The bimorph deflection is estimated using its radius of curvature, $r$ (Figure 2d), which depends on the stress from the temperature change $(\Delta T)$ and the residual stress that may be present in the material layers. The thermomechanical modeling for bimorph actuators is well documented based on bi-metal thermostats as

$$
r=\frac{\left[\left(E_{1} t_{1}^{2}\right)^{2}+\left(E_{2} t_{2}^{2}\right)^{2}+2 E_{1} E_{2} t_{1} t_{2}\left(2 t_{1}^{2}+3 t_{1} t_{2}+2 t_{2}^{2}\right)\right]}{\left[6 E_{1} E_{2} t_{1} t_{2}\left(t_{1}+t_{2}\right)\left(\alpha_{1}-\alpha_{2}\right) \Delta T\right]}
$$

where $t_{i}(i=1$ and 2) is the layer thickness of each layer $(1=$ PDMS and $2=\mathrm{PP}-\mathrm{PEDOT}$ ), and $E_{i}$ is the Young's modulus of the $i$ th layer. As the thickness of the PP-PEDOT layer is much smaller than that of PDMS, $t_{1} \gg t_{2}$, equation (1) can be simplified to

$$
r=\frac{\frac{E_{1} t_{1}^{2}}{6 E_{2} t_{2}}+\frac{2 t_{1}}{3}}{\left(\alpha_{1}-\alpha_{2}\right) \Delta T}
$$


Thus, $r$ decreases as $t_{1}$ and $E_{1}$ decrease. Furthermore, $r$ decreases as the difference in the CTE and temperature rise $(\Delta \mathrm{T})$ increases. The bending angle $(\theta)$ can be derived from the difference in the radius of the cured samples (Figure $2 \mathrm{~d}$ ) by assuming linear thermal expansion and the relationship between the radius and circumference as

$$
\theta=\frac{c \times \Delta L}{t_{1}}
$$

where $c$ is a correlation factor, including the angle conversion factor, and $\Delta L$ is the displacement difference between PP-PEDOT and PDMS due to the temperature evolution. From equations (1) and (4), the bending angle can be expressed as

$$
\theta=c \times\left(\alpha_{1}-\alpha_{2}\right) \times \Delta T \times \frac{L}{t_{1}}
$$

The relationship between the bending angle and the thickness of each layer is illustrated by the simulation plot in Figure 2e. The $t_{2}$ is significantly small compared to $t_{1}$, thus, the actuation properties of the bimorphs are mainly affected by the thickness of the PDMS, as shown in the simulated plot for a $\Delta \mathrm{T}$ of 10,20 and $90 \mathrm{~K}$ (Figure 2e), when the photothermal effect of PP-PEDOT was not considered in the simulation. However, the $\Delta \mathrm{T}$ from the photothermal effect of PPPEDOT is highly dependent on $t_{2}$, as shown in Figure $2 \mathrm{~b}$, so, it is critical to control $t_{2}$ to obtain high photothermal actuation. This is a productive way to optimize folding because increasing $t_{2}$ does not significantly affect to the total film thickness. Furthermore, it was encouraging that the simulated bending angle was large $\left(>90^{\circ}\right)$, which ensures effective folding of the bimorph under optimized conditions. To find an optimal bimorph for large photothermal folding, samples with different layer thicknesses were prepared. The sample name of the bimorph was abbreviated based on the thickness of the PP-PEDOT and PDMS layers in the micrometer scale. For instance, the bimorph with a $400 \mathrm{~nm}$-thick PEDOT layer and $70 \mu \mathrm{m}$-thick PDMS layer is abbreviated as PP0.4PD70.

The bimorphs deflected upward when the PP-PEDOT surface was on top and exposed to a NIR source, which is similar to a bending actuator or U-shaped memory, as shown for PP0.4PD70 and PP0.29PD70 (in Figure 3a, Supplementary Figure S3 and Supplementary Movie S1). ${ }^{31,52,53}$ In particular, PP0.4PD70 showed very fast folding within $2 \mathrm{~s}$ of NIR exposure $\left(I_{\mathrm{O}}=198 \mathrm{~mW}\right)$ and had a deflection angle larger than $90^{\circ}$. It returned to its original position (red mark) once the laser was turned off. Supplementary Figure S4 shows the correlation between the simulation result from equation (5) and the experimental bending angles under different light intensities for the bimorphs in this work. The simulation results matched well with the experimental values when the bending angle was $<45^{\circ}$ or the light intensity was lower than $100 \mathrm{~mW}$ with the $c$ value of the angle conversion factor $\left(360^{\circ} / 2 \pi\right)$. Considering the simulation results fit well with the experimental bending data, the photothermal energy in the PEDOT/PDMS bimorph seems to be mostly consumed by folding with negligible side reactions, such as distortion of the bimorph or inflation of the PDMS.

As expected from the equations, $\Delta T$ is crucial for folding (see the simulated plot in Figure 2e for $\Delta T=10,20$ and $90 \mathrm{~K}$ ). Figure $3 \mathrm{~b}$ shows the effect of $I_{\mathrm{o}}$ on the photoinduced displacement $\left(d_{\text {curv }}\right)$. It increases significantly as $I_{\mathrm{O}}$ increases, up to $102 \mathrm{~mW}$. The PP0.4PD70 bimorph showed a deflection angle $>120^{\circ}$ with a $d_{\text {curv }}$ of $20 \mathrm{~mm}$ within $2 \mathrm{~s}$ of NIR laser exposure $\left(I_{0}=198 \mathrm{~mW}\right)$ and flipped over when exposed to a laser with a higher $I_{\mathrm{O}}(>102 \mathrm{~mW})$. Other samples showed almost linear increases in $d_{\text {curv }}$ as $I_{\mathrm{o}}$ increased up to $198 \mathrm{~mW}$. The maximum displacement sensitivity $\left(S_{\mathrm{dL}}\right)$ was determined using the slope of the plot in Figure $3 \mathrm{~b}$ and was 0.2 and $0.087 \mathrm{~mm} \mathrm{~mW}^{-1}$ for PP0.4D70 and PP0.29D70, respectively. As shown in Figure 3c, $S_{\mathrm{dL}}$ increased with the increasing PP-PEDOT thickness $\left(t_{2}\right)$ due to the increase in the photothermally generated heat. On the other hand, $S_{\mathrm{dL}}$ decreased by increasing the PDMS thickness $\left(t_{1}\right)$. Considering the differences in the CTE and Young's modulus for each layer in the bimorphs (Supplementary Figure S2b), a thin PDMS layer and thick PEDOT layer are the best options to obtain the maximum folding under a low light intensity. However, the PDMS thickness was limited due to the dimensional instability of the bimorphs with $t_{1}<70 \mu \mathrm{m}$. The $400 \mathrm{~nm}$-thick PP-PEDOT film absorbed more than $90 \%$ of the $808 \mathrm{~nm}$ NIR light, as shown in Figure 2a, which indicated that the optimized bimorph was PP0.4PD70 within these experimental conditions.

The photothermal bimorph was highly reversible, and the folded strip returned to its original form (Supplementary Figure S3). Furthermore, the displacement was almost constant as shown for PP0.4PD70 under NIR exposure $\left(I_{\mathrm{O}}=57.5 \mathrm{~mW}\right)$ in Figure 3d. The loss of displacement $(\delta \Delta L)$ under the cycle experiments was $<1 \%$ under the exposure of a $102 \mathrm{~mW}$ laser with a repetitive pulse $(3 \mathrm{~s}$ on and $12 \mathrm{~s}$ off), as shown in Supplementary Figure S5a. To speed up the displacement loss or bimorph failure, we carried out cycle experiments under an extreme condition with a high-power laser $\left(I_{\mathrm{O}}=198 \mathrm{~mW}\right)$. As shown in Supplementary Figure S5b, the photothermal folding was highly reversible and the $\delta \Delta L$ value at the 9000th cycle was $8.4 \%$, which is a promising result. After optimization of the sample, it could be applied to real applications.

\section{Photothermal folding and generation of complex 3D structures}

Taking advantage of their flexibility and cuttability, the PP-PEDOT/ PDMS bimorphs were generated in various shapes. Supplementary Figure S6 shows the worm-like walking of the PP0.29PD70 bimorph as it moved forward. This caused the actuator to bend repeatedly and move in the opposite direction. The displacement over $10 \mathrm{~s}$ was $3 \mathrm{~mm}$ from one irradiation cycle with a movement speed of $3.0 \times 10^{-4} \mathrm{~m} \mathrm{~s}^{-1}$, which is comparable to or even greater than the reported value. ${ }^{54,55}$ As the PP-PEDOT/PDMS bimorphs are waterinsoluble, they can float and swim to specific locations in water using remotely controlled NIR light. A biomimetic microfish actuator (18 $\mathrm{mm}$ in length and $6 \mathrm{~mm}$ in width) was fabricated to mimic fish swimming in water. The force exerted on the water from such motion laterally cancels. However, it generates a net backward force from the photothermal actuation, and the fish moves forward through the water. The required energy for the fish movement in this work $\left(I_{\mathrm{o}}=1.1 \mathrm{~W}, 2.94 \mathrm{~W} \mathrm{~cm}^{-2}\right)$ was less than that of the previously reported fish-like platforms made of soft materials $\left(29.5 \mathrm{~W} \mathrm{~cm}^{-2}\right){ }^{1}$ The PP-PEDOT/PDMS bimorphs showed large movement $(>10 \mathrm{~cm})$ in water under a NIR laser $\left(I_{0}=253 \mathrm{~mW}\right.$; Figure 4a). This is promising for applications in remote actuation. As summarized in Supplementary Table S2, the displacement and bending angles of the PP-PEDOT/PDMS bimorphs were the largest among the PEDOTbased actuators, including electrothermal, piezoelectric and hydrogel (Supplementary Figure S7).

As the folding of the PP-PEDOT/PDMS bimorph is large with a deflection angle of $>90^{\circ}$, a programmable $2 \mathrm{D}$ array was explored to reversibly generate complex $3 \mathrm{D}$ architectures in response to NIR light irradiation. A 2D array with a sunflower-like structure (Figure $4 \mathrm{~b}$ ) was prepared by tailoring a large-area bimorph film with a cutter. The $2 \mathrm{D}$ sunflowers A and B consisted of 13 and 16 flat bilayer lobes, respectively, that were connected at the center (diameters $=4$ and $5 \mathrm{~mm}$, respectively). Upon exposure to one sun (AM1.5, 
a
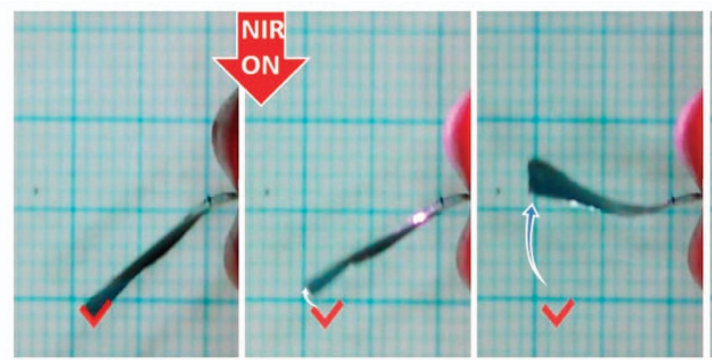

b

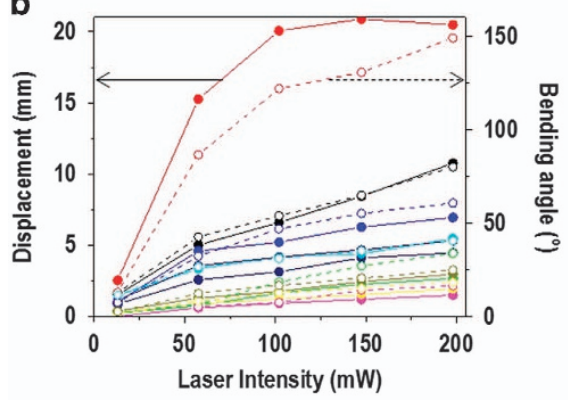

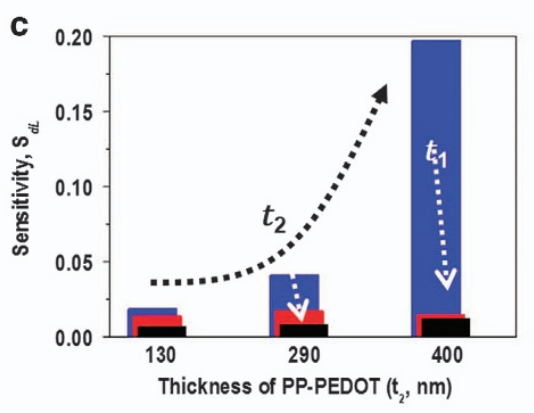

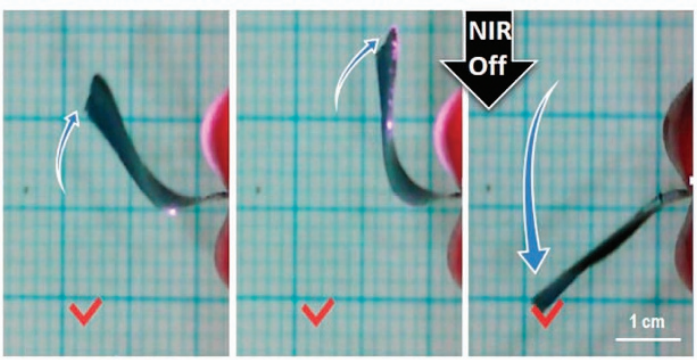

d

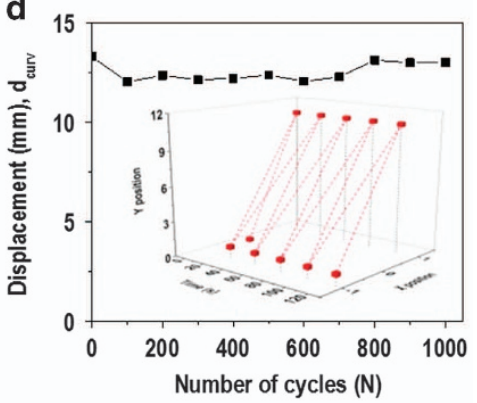

Figure 3 (a) Reversible folding of PPO.4PD70 via near-infrared (NIR) light (198 mW). The red mark indicates the original position before NIR irradiation. (b) Photothermally induced displacement ( $d_{\text {curv, }}$ solid) and bending angle (empty, dash) of the bimorphs under different laser intensities with different thicknesses of the PP-PEDOT and poly(dimethylsiloxane) (PDMS) layers for PP0.40PD70 (red), PP0.29PD70 (black), PP0.13PD70 (navy), PP0.40PD160 (cyan), PP0.29PD160 (blue), PP0.13PD160 (green), PPO.40PD300 (dark yellow), PP0.29PD300 (yellow) and PP0.13PD300 (magenta). (c) Displacement sensitivity of the bimorphs with different PP-PEDOT $\left(t_{2}\right)$ and PDMS ( $\left.t_{1}\right)$ layer thicknesses, where $t_{1}$ varies from $70 \mu \mathrm{m}$ (blue) to $160 \mathrm{~nm}$ (red) and $300 \mathrm{~nm}$ (black). (d) Cyclability of photothermal actuation for PP0.4PD70 under a $57.5 \mathrm{~mW}$ NIR laser over 1000 cycles. The inset graph shows the three-dimensional cyclability. PEDOT, poly(3,4-ethylenedioxythiophene).

$100 \mathrm{~mW} \mathrm{~cm}^{-2}$ ), sunflower B heated, folded up to $45^{\circ}$ within $10 \mathrm{~s}$ and converted into a $3 \mathrm{D}$ flower structure. It further folded within $15 \mathrm{~s}$ of one sun exposure (Supplementary Figure S8). The folded 3D structure returned to the original $2 \mathrm{D}$ sunflower when the light source was turned off. Under strong NIR laser irradiation, however, the photothermal folding was faster. Sunflower B folded within $2 \mathrm{~s}$ and further folded to generate an artificial Venus flytrap within $5 \mathrm{~s}$ of NIR exposure (Figure 4c and Supplementary Movie S2). When the light was turned off, the flytrap returned to its original 2D structure. From the thermal image, the heating and cooling matched the conversion of the $2 \mathrm{D}$ structure into the $3 \mathrm{D}$ structure or vice versa, as shown in Figure $4 \mathrm{~d}$. Interestingly, the heat development mirrored the folding structure and generated a heat pocket inside the flytrap (Figure $4 \mathrm{~d}$, iii). The thermal conductivity $(k)$ of PDMS is $0.15 \mathrm{~W} \mathrm{~m}^{-2.56}$ Because the developed heat was protected by the PDMS layer, which has a low $k$, heat builds up inside the pocket. The temperature of the trap was over $100{ }^{\circ} \mathrm{C}$, which may be useful for a wide range of chemical and physical heat transformations in $3 \mathrm{D}$. This indicates that the bimorphic $2 \mathrm{D}$ structure can be used to reversibly generate a heat pocket to localize heat inside a 3D structure. NIR can transmit through PDMS and various organisms and tissues, thus, it can be useful for programming bendable or imbedded electronics. Owing to the reversible foldability of the Venus flytrap, it can be used for trapping and moving objects like an excavator. Sunflower A was connected to a bar, and an object was tested for trapping (Supplementary Movie S3). When sunflower A was placed on the sponge under NIR exposure $\left(I_{\mathrm{O}}=1.1 \mathrm{~W}\right.$, $910 \mathrm{~mW} \mathrm{~cm}^{-2}$ ), it folded, converted into an excavator and trapped the sponge ball. The ball was replaced on the target location when the excavator released it upon cooling. Figure $4 \mathrm{e}$ shows the $1 \mathrm{~cm}$ distance movement of a sponge by the excavator. These results suggest that the bimorphs with PP-PEDOTs and PDMS offer effective photothermal folding and generation of a complex biomimetic trap on command.

\section{CONCLUSION}

In summary, PP-PEDOT/PDMS bimorphs were prepared via a dry transfer method to afford a soft bimorph with unique NIR lightresponsive characteristics. The reversible folding and actuation properties were optimized by controlling the film thicknesses of each layer and the radiation laser intensity. The photothermal folding was modeled according to the bimetallic actuator, as summarized in equations (1)-(5). The deflection and bending angles of the PPPEDOT/PDMS bimorphs were inversely increased by the thickness of the PDMS layer $\left(t_{1}\right)$ because $t_{1}$ is much larger than that of PP-PEDOT, as shown in Figure 2e. Although the thickness of PP-PEDOT $\left(t_{2}\right)$ is negligible compared to the total thickness $\left(t_{1}+t_{2}\right)$ of the bimorph, the effect of $t_{2}$ on the photothermal folding was critical because the thicker PP-PEDOT layer affords a higher temperature increase upon NIR exposure (Figure $2 \mathrm{~b}$ and $\mathrm{c}$ ). Thus, $t_{2}$ was maximized at $400 \mathrm{~nm}$ and $t_{1}$ was minimized at $70 \mu \mathrm{m}$ to obtain high folding with a displacement $\left(d_{\text {curv }}\right)$ of up to $20 \mathrm{~mm}$ and a deflection angle of up to $150^{\circ}$ (Figure $3 \mathrm{~b}$ ). Supplementary Table S2 and Supplementary Figure S7 show that the displacement and bending properties of the PP-PEDOT/PDMS bimorphs in this study are the largest among other reported soft actuators based on PEDOT, including electrothermal, piezoelectric and photothermal actuators. Such a large actuation from the PP-PEDOT/ PDMS bilayer paves the way for the reversible formation of a foldable structure via a photothermal process. Potential applications for the photothermal PP-PEDOT/PDMS bimorphs were demonstrated via experiments in air and water media. The movement can be remotely controlled using NIR light irradiation. Together with the photoactuation, the large displacement via NIR exposure could lead to a programmable bimorph that can generate complex chaotic architectures, such as a Venus flytrap and an excavator, that are useful for a broad range of biomimetic applications. Furthermore, the flat $2 \mathrm{D}$ structure can be used for the reversible generation of a heat pocket or 
a
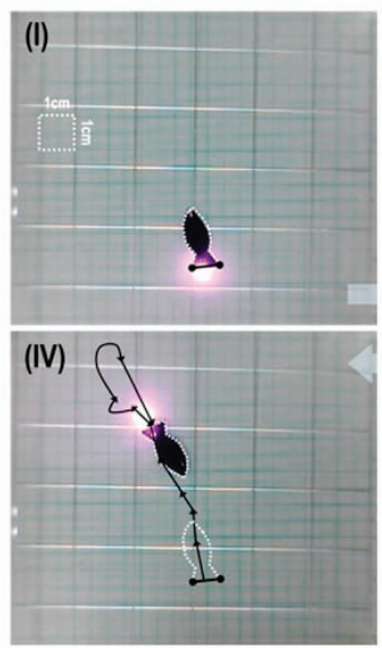

C

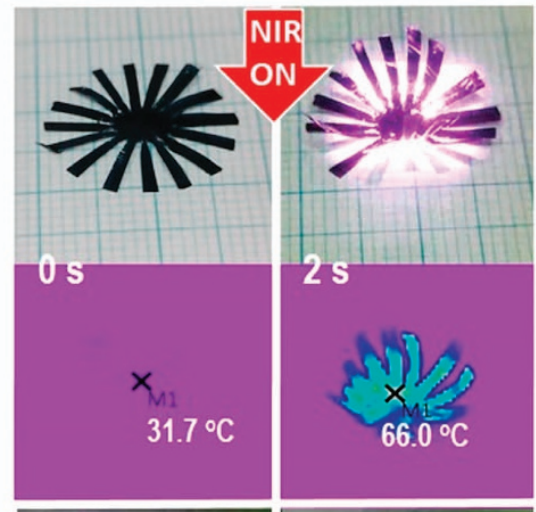

e
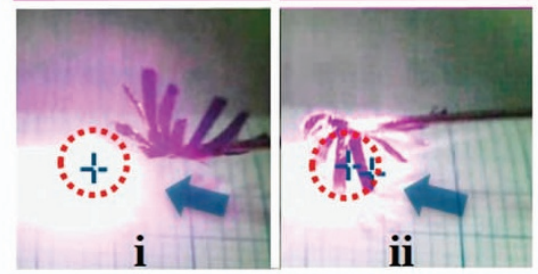
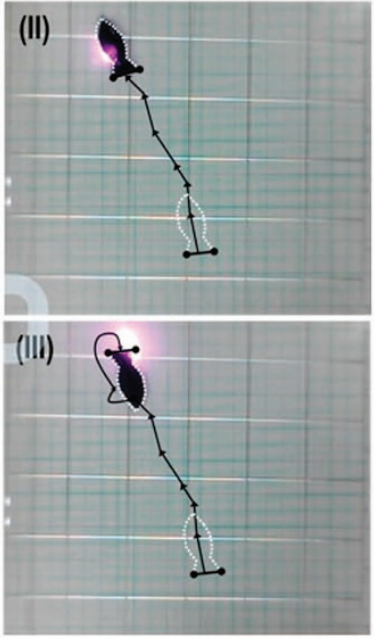

b

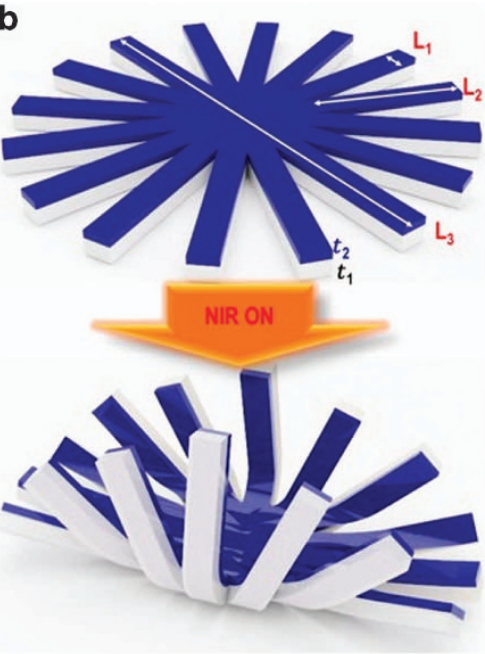

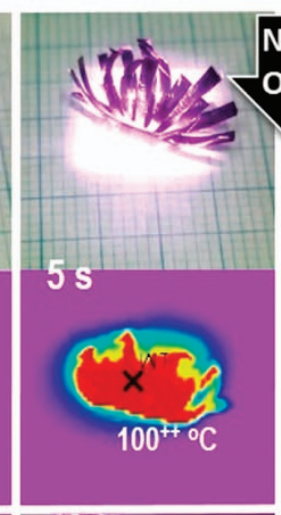
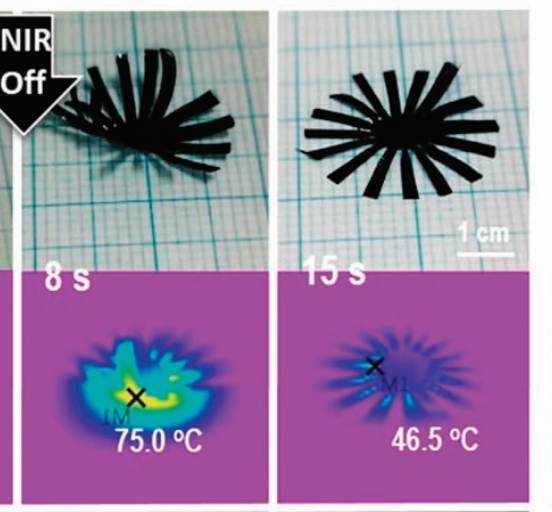

$100^{\circ} \mathrm{C}$
-1000
-500
-000
-700
-600
-500
-40.0
-300
-500
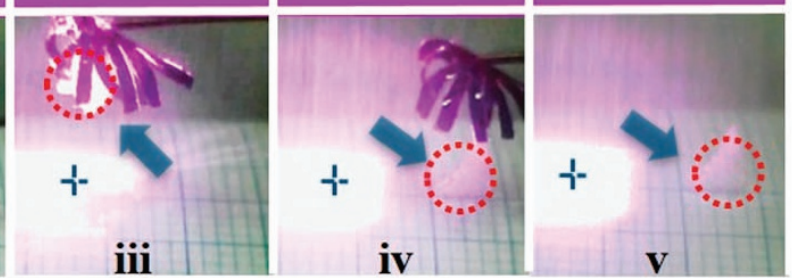

Figure 4 (a) Photographic images of the photothermal movement of a microfish made from PP0.40PD70, on water (under $253 \mathrm{~mW}$ ). (b) Schematic images of the formation of a Venus flytrap from PP-PEDOT $\left(t_{2}=400 \mathrm{~nm}\right) /$ poly(dimethylsiloxane) (PDMS; $\left.t_{1}=70 \mu \mathrm{m}\right)$ bimorphs: the sizes $\left(L_{1}, L_{2}\right.$ and $\left.L_{3}\right)$ of sunflowers $A$ and $B$ were $12 \mathrm{~mm}, 4 \mathrm{~mm}, 0.8 \mathrm{~mm}$ and $17 \mathrm{~mm}, 6 \mathrm{~mm}, 1 \mathrm{~mm}$, respectively. (c) Photographic image and (d) thermal image of the formation of a Venus flytrap from sunflower B via near-infrared (NIR) irradiation (1.1 W). (e) Photographic image of the trapping and moving of a sponge via the formation of an excavator from sunflower A via NIR irradiation (1.1 W). PEDOT, poly(3,4-ethylenedioxythiophene).

heat trap to localize heat inside a 3D structure. Thus, photothermal heating can be effective not only in a planar biomorph but also in a curved pocket-like structure because NIR is transmitted into the thick PDMS layer to stimulate the inner PP-PEDOT layer, and the developed heat is trapped by the polymer pocket with a low $k$. NIR can transmit through PDMS and various organisms and tissues; thus, it is useful for programming bendable or imbedded electronics. Therefore, it is anticipated that the water-insoluble PP-PEDOT/PDMS bimorph will provide a novel platform for light-driven motors, oscillators, light sensors and wireless actuators controlled using a NIR source, and used to explore biomimetic motion in ambient and aqueous media.

\section{CONFLICT OF INTEREST}

The authors declare no conflict of interest.

\section{ACKNOWLEDGEMENTS}

We thank Paul Ahre at LLNL for fabricating the SMP coil mold. We acknowledge financial support from a National Research Foundation (NRF) grant funded by the Korean government (Ministry of Science, ICT \& Future Planning, MSIP) through the Global Research Lab. (GRL: 2016K1A1A2912753).

1 Jiang, W., Niu, D., Liu, H., Wang, C., Zhao, T., Yin, L., Shi, Y., Chen, B., Ding, Y. \& Lu, B. Photoresponsive soft-robotic platform: biomimetic fabrication and remote actuation. Adv. Funct. Mater. 24, 7598-7604 (2014).

2 Kawahara, T., Sugita, M., Hagiwara, M., Arai, F., Kawano, H., Shihira-Ishikawa, I. \& Miyawaki, A. On-chip microrobot for investigating the response of aquatic microorganisms to mechanical stimulation. Lab Chip 13, 1070-1078 (2013).

3 Brochu, P. \& Pei, Q. Advances in dielectric elastomers for actuators and artificial muscles. Macromol. Rapid Commun. 31, 10-36 (2010).

4 Halsey, T. Electrorheological fluids. Science 258, 761-766 (1992).

5 Ilievski, F., Mazzeo, A. D., Shepherd, R. F., Chen, X. \& Whitesides, G. M. Soft robotics for chemists. Angew. Chem. Int. Ed. 123, 1930-1935 (2011).

6 Otsuka, K. \& Ren, X. Physical metallurgy of Ti-Ni-based shape memory alloys. Prog. Mater. Sci. 50, 511-678 (2005).

7 Wu, C., Feng, J., Peng, L., Ni, Y., Liang, H., He, L. \& Xie, Y. Large-area graphene realizing ultrasensitive photothermal actuator with high transparency: new prototype robotic motions under infrared-light stimuli. J. Mater. Chem. 21, 18584-18591 (2011). 
8 Welty, R. J., Mochizuki, K., Lutz, C. R., Welser, R. E. \& Asbeck, P. M. Design and performance of tunnel collector HBTs for microwave power amplifiers. IEEE Trans. Electron. Devices 50, 894-900 (2003).

9 Elbuken, C., Gui, L., Ren, C. L., Yavuz, M. \& Khamesee, M. B. Design and analysis of a polymeric photo-thermal microactuator. Sens. Actuator A Phys. 147, 292-299 (2008).

10 Zhang, X., Yu, Z., Wang, C., Zarrouk, D., Seo, J.-W. T., Cheng, J. C., Buchan, A. D., Takei, K., Zhao, Y. \& Ager, J. W. Photoactuators and motors based on carbon nanotubes with selective chirality distributions. Nat. Commun. 5, 2983-2983 (2014).

11 Taccola, S., Greco, F., Sinibaldi, E., Mondini, A., Mazzolai, B. \& Mattoli, V. Toward a new generation of electrically controllable hygromorphic soft actuators. Adv. Mater. 27, 1668-1675 (2015)

12 Kim, U., Kang, J., Lee, C., Kwon, H. Y., Hwang, S., Moon, H., Koo, J. C., Nam, J.-D., Hong, B. H. \& Choi, J.-B. A transparent and stretchable graphene-based actuator for tactile display. Nanotechnology 24, 145501 (2013).

13 Zhu, S.-E., Shabani, R., Rho, J., Kim, Y., Hong, B. H., Ahn, J.-H. \& Cho, H. J. Graphene-based bimorph microactuators. Nano Lett. 11, 977-981 (2011).

14 Xie, X., Qu, L., Zhou, C., Li, Y., Zhu, J., Bai, H., Shi, G. \& Dai, L. An asymmetrically surface-modified graphene film electrochemical actuator. ACS Nano $\mathbf{4}$, 6050-6054 (2010).

15 Jager, E. W., Smela, E. \& Inganäs, O. Microfabricating conjugated polymer actuators. Science 290, 1540-1545 (2000).

16 Otero, T. F. Biomimetic conducting polymers: synthesis, materials, properties, functions, and devices. Polym. Rev. 53, 311-351 (2013).

17 Sansinena, J., Olazabal, V., Otero, T., Da Fonseca, C. P. \& De Paoli, M.-A. A solid state artificial muscle based on polypyrrole and a solid polymeric electrolyte working in air. Chem. Commun. 22, 2217-2218 (1997).

$18 \mathrm{Wu}$, Y., Alici, G., Spinks, G. M. \& Wallace, G. Fast trilayer polypyrrole bending actuators for high speed applications. Synth. Met. 156, 1017-1022 (2006).

19 Okuzaki, H., Kuwabara, T., Funasaka, K. \& Saido, T. Humidity-sensitive polypyrrole films for electro-active polymer actuators. Adv. Funct. Mater. 23, 4400-4407 (2013).

20 Carpi, F. \& Smela, E. Biomedical Applications of Electroactive Polymer Actuators, Wiley: UK, (2009).

21 Kim, B., Shin, H., Park, T., Lim, H. \& Kim, E. NIR-sensitive poly (3, 4-ethylenedioxyselenophene) derivatives for transparent photo-thermo-electric converters. Adv. Mater. 25, 5483-5489 (2013).

22 Park, T., Na, J., Kim, B., Kim, Y., Shin, H. \& Kim, E. Photothermally activated pyroelectric polymer films for harvesting of solar heat with a hybrid energy cell structure. ACS Nano 9, 11830-11839 (2015).

23 Wu, Z., Lin, X., Wu, Y., Si, T., Sun, J. \& He, Q. Near-infrared light-triggered 'on/off' motion of polymer multilayer rockets. ACS Nano 8, 6097-6105 (2014).

24 You, J., Heo, J. S., Kim, J., Park, T., Kim, B., Kim, H.-S., Choi, Y., Kim, H. O. \& Kim, E. Noninvasive photodetachment of stem cells on tunable conductive polymer nano thin films: selective harvesting and preserved differentiation capacity. ACS Nano 7 , 4119-4128 (2013).

25 Kim, J. D., Heo, J. S., Park, T., Park, C., Kim, H. O. \& Kim, E. Photothermally induced local dissociation of collagens for harvesting of cell sheets. Angew. Chem. Int. Ed. 127, 5967-5971 (2015)

26 Kim, J., Lee, E., Hong, Y., Kim, B., Ku, M., Heo, D., Choi, J., Na, J., You, J. \& Haam, S. Self-doped conjugated polymeric nanoassembly by simplified process for optical cancer theragnosis. Adv. Funct. Mater. 25, 2260-2269 (2015).

27 Lee, C. H., Kim, J.-H., Zou, C., Cho, I. S., Weisse, J. M., Nemeth, W., Wang, Q., Van Duin, A. C., Kim, T.-S. \& Zheng, X. Peel-and-stick: mechanism study for efficient fabrication of flexible/transparent thin-film electronics. Sci. Rep. 3, 2917 (2013).

28 Park, T., Kim, B., Kim, Y. \& Kim, E. Highly conductive PEDOT electrodes for harvesting dynamic energy through piezoelectric conversion. J. Mater. Chem. A 2, 5462-5469 (2014).

29 Khadka, R. \& Yim, J.-H. Influence of base inhibitor and surfactant on the electrical and physicochemical properties of PEDOT-SiO2 hybrid conductive films. Macromol. Res. 23, 559-565 (2015).

30 Li, Q., Liu, C., Lin, Y.-H., Liu, L., Jiang, K. \& Fan, S. Large-strain, multiform movements from designable electrothermal actuators based on large highly anisotropic carbon nanotube sheets. ACS Nano 9, 409-418 (2015).

31 Chen, L., Liu, C., Liu, K., Meng, C., Hu, C., Wang, J. \& Fan, S. High-performance, lowvoltage, and easy-operable bending actuator based on aligned carbon nanotube/polymer composites. ACS Nano 5, 1588-1593 (2011).

32 Cha, S., Cha, M., Lee, S., Kang, J. H. \& Kim, C. Low-temperature, dry transfer-printing of a patterned graphene monolayer. Sci. Rep. 5, 17877 (2015).

33 Carlson, A., Bowen, A. M., Huang, Y., Nuzzo, R. G. \& Rogers, J. A. Transfer printing techniques for materials assembly and micro/nanodevice fabrication. Adv. Mater. 24, 5284-5318 (2012).

34 Armani, D., Liu, C. \& Aluru, N. in MEMS'99 Twelfth IEEE International Conference on 222-227 IEEE, 1999.
35 Kuila, B. K. \& Nandi, A. K. Structural hierarchy in melt-processed poly (3-hexyl thiophene)-montmorillonite clay nanocomposites: novel physical, mechanical, optical, and conductivity properties. J. Phys. Chem. B 110, 1621-1631 (2006).

36 Miyauchi, S., Kondo, T., Oshima, K., Yamauchi, T., Shimomura, M. \& Mitomo, H. Electrical and mechanical characteristics of composites consisting of fractionated poly (3-hexylthiophene) and conducting particles. J. Appl. Polym. Sci. 85, 1429-1433 (2002)

37 Woo, W., Feng, Z. L., Wang, X., Brown, D. W., Clausen, B., Hubbard, C., Choo, H. \& David, S. Quasi-Steady state principle and in situ real-time investigation of transient strains in 6061-T6 Al alloy using neutron diffraction. Key Eng. Mater. 345, 797-800 (2007).

38 Balagaddé, F. K., You, L., Hansen, C. L., Arnold, F. H. \& Quake, S. R. Long-term monitoring of bacteria undergoing programmed population control in a microchemostat. Science 309, 137-140 (2005).

39 Koschwanez, J. H., Carlson, R. H. \& Meldrum, D. R. Thin PDMS films using long spin times or tert-butyl alcohol as a solvent. PLOS ONE 4, e4572 (2009).

40 Lee, S. W. \& Lee, S. S. Shrinkage ratio of PDMS and its alignment method for the wafer level process. Microsys. Technol. 14, 205-208 (2008).

41 Szczepanski, C. R., Darmanin, T. \& Guittard, F. Spontaneous, phase-separation induced surface roughness: a new method to design parahydrophobic polymer coatings with rose petal-like morphology. ACS Appl. Mater. Interfaces 8, 3063-3071 (2016).

42 Smits, F. Measurement of sheet resistivities with the four-point probe. Bell Labs Techn. J. 37, 711-718 (1958).

43 Park, T., Park, C., Kim, B., Shin, H. \& Kim, E. Flexible PEDOT electrodes with large thermoelectric power factors to generate electricity by the touch of fingertips. Energy Environ. Sci. 6, 788-792 (2013).

44 Zhang, X. D., Wang, H., Antaris, A. L., Li, L., Diao, S., Ma, R., Nguyen, A., Hong, G., Ma, Z. \& Wang, J. Traumatic brain injury imaging in the second near-infrared window with a molecular fluorophore. Adv. Mater. 28, 6872-6879 (2016).

45 Diao, S., Blackburn, J. L., Hong, G., Antaris, A. L., Chang, J., Wu, J. Z., Zhang, B., Cheng, K., Kuo, C. J. \& Dai, H. Fluorescence imaging in vivo at wavelengths beyond 1500 nm. Angew. Chem. Int. Ed. 127, 14971-14975 (2015).

46 Lin, Y.-H., Kang, S.-W. \& Wu, T.-Y. Fabrication of polydimethylsiloxane (PDMS) pulsating heat pipe. Appl. Therm. Eng. 29, 573-580 (2009).

47 Imai, S. \& Adachi, K. Fundamental characteristics of a MEMS-diaphragm actuator using a thermal expansion drive composed of a conductive polymer. IEEJ Trans. Electr. Electron. Eng. 10, 101-108 (2015).

48 Peng, W., Xiao, Z. \& Farmer, K. Optimization of thermally actuated bimorph cantilevers for maximum deflection. Nanotech. Proc. 1, 376-379 (2003).

49 Timoshenko, S. Analysis of bi-metal thermostats. J. Opt. Soc. Am. B Opt. Phys. 11, 233-255 (1925).

50 Todd, S. T. Electrothermomechanical Modeling of a 1-D Electrothermal MEMS Micromirror. (MSc thesis, Univ. Florida, 2005).

51 Wang, Z. Polydimethylsiloxane mechanical properties measured by macroscopic compression and nanoindentation techniques (2011) http://scholarcommons.usf.edu/ etd/3402/.

52 Leng, J., Huang, W., Lan, X., Liu, Y. \& Du, S. Significantly reducing electrical resistivity by forming conductive Ni chains in a polyurethane shape-memory polymer/carbon-black composite. Appl. Phys. Lett. 92, 204101 (2008).

53 Luo, X. \& Mather, P. T. Conductive shape memory nanocomposites for high speed electrical actuation. Soft Matter 6, 2146-2149 (2010).

54 Cho, M., Seo, H., Nam, J., Choi, H., Koo, J., Song, K. \& Lee, Y. A solid state actuator based on the PEDOT/NBR system. Sens. Actuator B Chem. 119, 621-624 (2006).

55 Zainudeen, U. L., Careem, M. A. \& Skaarup, S. PEDOT and PPy conducting polymer bilayer and trilayer actuators. Sens. Actuator B Chem. 134, 467-470 (2008).

56 Mark, J. E. Polymer Data Handbook (Oxford Univ. Press, Oxford, England, 2009).

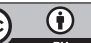

This work is licensed under a Creative Commons Attribution 4.0 International License. The images or other third party material in this article are included in the article's Creative Commons license, unless indicated otherwise in the credit line; if the material is not included under the Creative Commons license, users will need to obtain permission from the license holder to reproduce the material. To view a copy of this license, visit http:// creativecommons.org/licenses/by/4.0/

(C) The Author(s) 2017

Supplementary Information accompanies the paper on the NPG Asia Materials website (http://www.nature.com/am) 